Jose M. Carnate Jr., MD

Department of Pathology College of Medicine - Philippine General Hospital University of the Philippines Manila
Correspondence: Jose M. Carnate, Jr. MD University of the Philippines Manila College of Medicine

Department of Pathology

547 Pedro Gil St., Ermita, Manila 1000

Philippines

Phone (632) 5264550

Fax (632) 4003638

Email:jmcjpath@yahoo.com

Reprints will not be available from the author.

\section{A Rare Case of Hoarseness: Laryngeal Amyloidosis}

This is the case of a 72-year-old male who presented with a three year history of hoarseness. Two years prior to admission, he developed dyspnea, dysphagia and odynophagia. Three months prior to admission, the patient visited a private physician who noted a $3 \mathrm{~cm}$ diameter ill-defined laryngeal thickening and advised biopsy, hence the present consult. A biopsy and subsequent hemilaryngectomy were performed.

Histologic sections from the mass show an abundant subepithelial deposition of an acellular, extracellular, eosinophilic and non-fibrillar matrix that surrounds atrophic mucous acini (Figuress. 1, 2, 3). Scattered lymphocytes and foreign-body-type giant cells are noted. Congo-red histochemical stains show a characteristic salmon-pink reaction (Figure 4) which on polarizing microscopy showed a characteristic apple-green birefringence. No evidence of malignancy is seen. The diagnosis was localized laryngeal amyloidosis.

Laryngeal amyloidosis is rare, accounting for less than $1 \%$ of benign laryngeal tumors. Most are localized - not associated with deposits elsewhere in the body and are primary - not associated with any known systemic or neoplastic diseases such as disseminated tuberculosis or certain lymphomas and multiple myeloma. Adults are usually affected although rarely, children can develop the condition. Almost all patients present with hoarseness, in addition to symptoms related to the size, extent and location of the deposits. The false vocal cord is often affected although multifocality within the larynx occurs in $15 \%$ of cases. ${ }^{1,3}$

Careful histologic evaluation should exclude the presence of a coexistent laryngeal condition, particularly the malignant neoplasms stated above, as these would require more extensive management. Other differential diagnoses include vocal cord polyps and papillomas. ${ }^{2,3}$

The prognosis for isolated laryngeal amyloidosis is excellent although occasional repeat surgeries may be necessary for recurrent disease. On the other hand, the prognosis of laryngeal amyloidosis associated with systemic or neoplastic conditions is dictated largely by the course of the coexisting disease processes. ${ }^{1}$

Although rare, laryngeal amyloidosis should not be overlooked in the differential diagnosis of hoarseness particularly in adults. An attempt to exclude a co-existing systemic or neoplastic disease should always be made. 


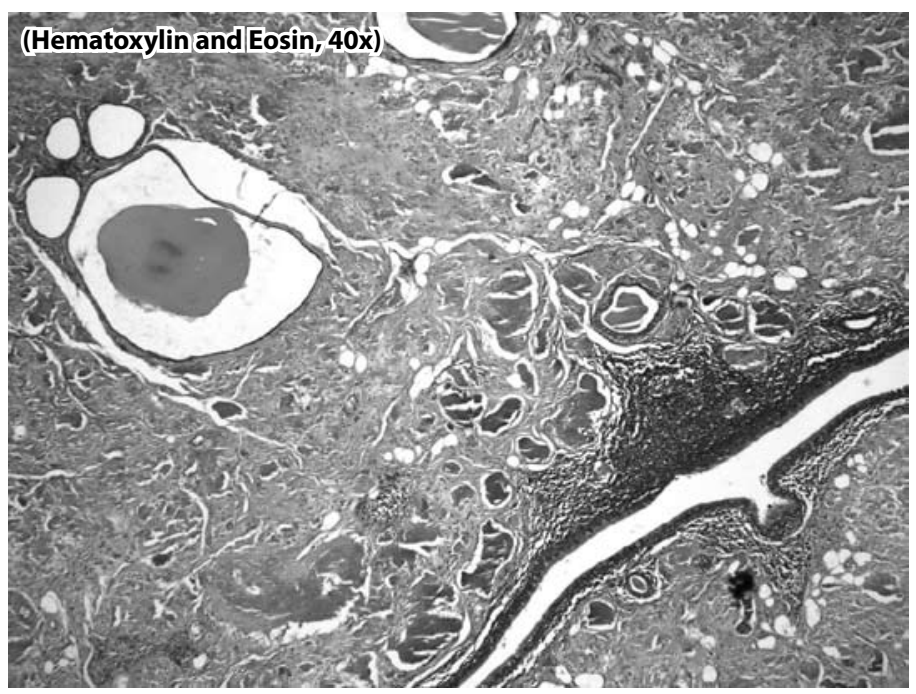

Figure 1. Extensive eosinophilic extracellular amorphous matrix (Hematoxylin and Eosin, 40x)

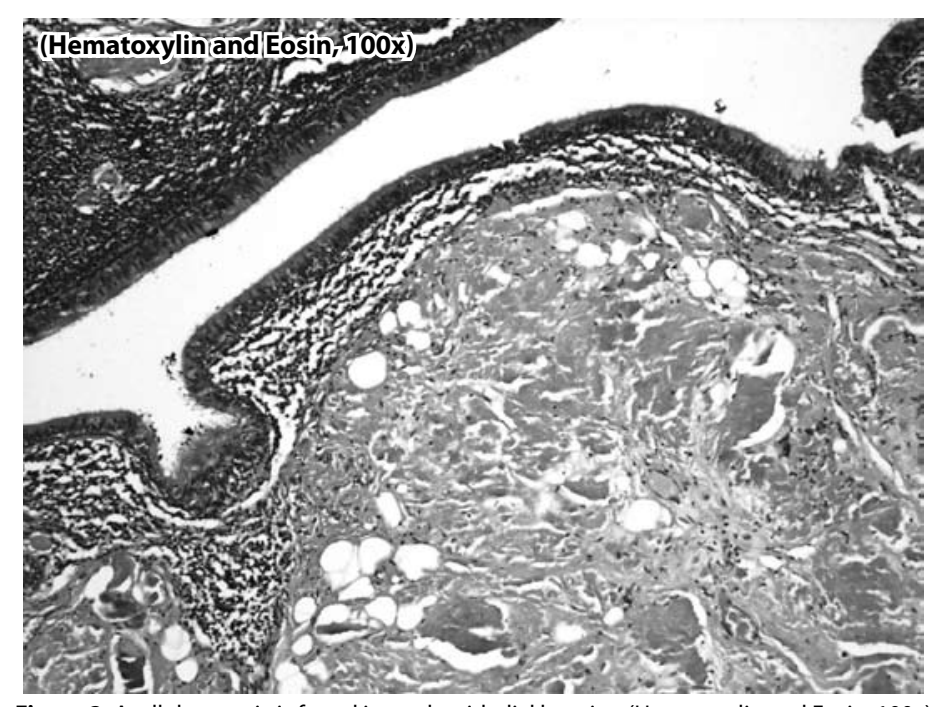

Figure 2. Acellular matrix is found in a subepithelial location (Hematoxylin and Eosin, 100x)

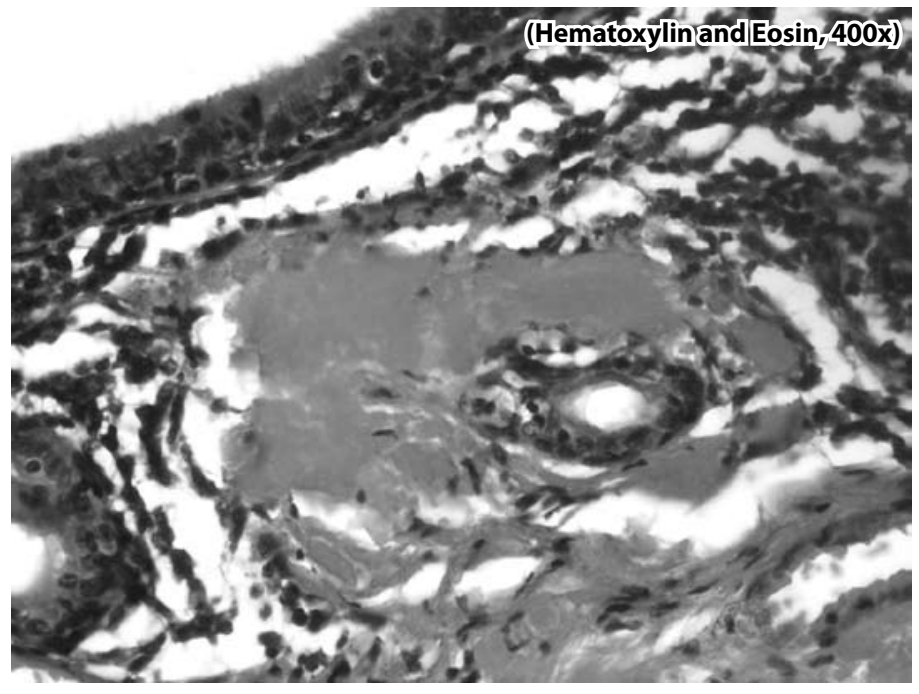

Figure 3. Matrix may have scattered lymphocytes and surround acini that undergo compressive atrophy (Hematoxylin and Eosin, 400x)

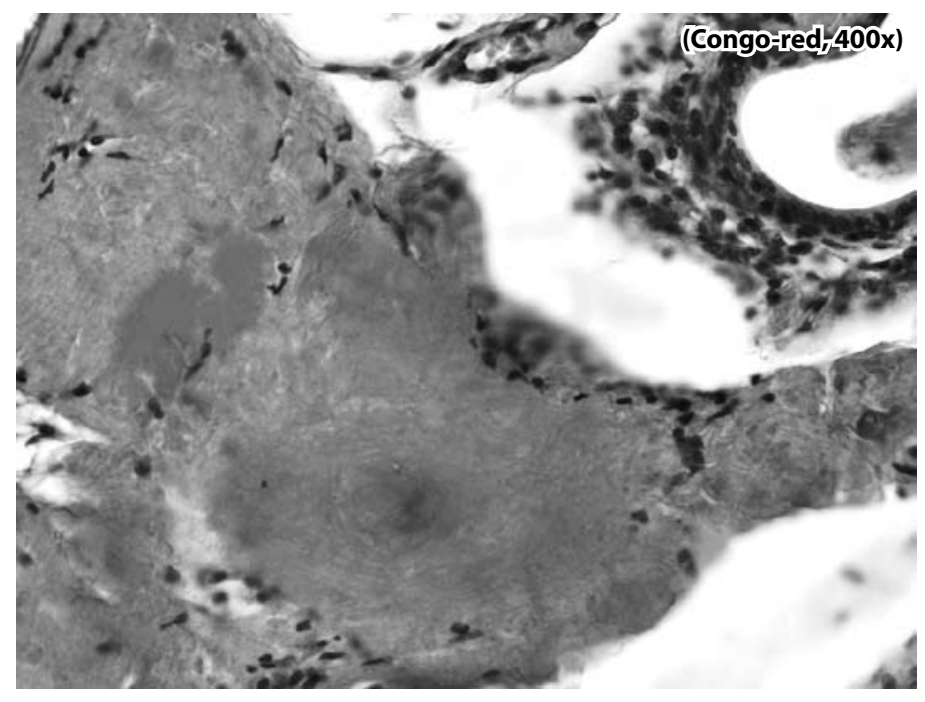

Figure 4. Characteristic salmon-pink reaction (Congo-red, 400x)

\section{REFERENCES}

1. Thompson LD. Head and Neck Pathology. In Foundations in Diagnostic Pathology Series. Goldblum JR ed. Churchill Livingstone Elsevier, Inc. 2006.

2. Wenig B. Atlas of Head and Neck Pathology, $2^{\text {nd }}$ ed. Elsevier, Inc. 2008.

3. Gnepp DR, ed. Diagnostic Surgical Pathology of the Head and Neck. WB Saunders Company, 2010. 